\title{
The Effects of Core Training on High School Baseball Performance
}

\author{
By Clint William Felion ${ }^{ \pm} \&$ Mark DeBeliso*
}

\begin{abstract}
Introduction: Core stability and core resistance training $(R T)$ have recently received a lot of attention for improving functional movement and athletic performance. Core strength as it relates to sports performance is the ability to transfer energy through the core during rapid, discrete motor skills. Purpose: The purpose of this study was to explore the effectiveness of a RT program that targets the muscles of the core in order to increase force production in torsional movements such as throwing and hitting in baseball. Methods: Twenty-four male high school (HS) baseball players participated in a six week, off-season, core RT program. The participants were randomly assigned to a control group that attended baseball-specific workouts twice a week for 6-weeks that included throwing, pitching, and hitting (CG: $n=12)$ or the core RT group (RTG: $n=12)$. The RTG participated in a 6-week core RT program (Pallof press, oblique twists, figure 8 's, standing medicine ball toss) in addition to baseball-specific practice twice a week. Throwing velocity (TV) and Ball-Exit Velocity (BEV) were assessed prior to and following the 6-week study intervention with a Stalker Sport II radar gun (mph). BEV was the speed of the ball immediately after being struck by the baseball bat. The dependent variables were compared within each group with dependent T-tests. A gain score was also calculated for each dependent variable and compared between the RTG and the CG with independent T-tests $(\alpha<0.05)$. Results: Neither the $C G$ nor the $R T G$ experienced an increase in $T V$ following the 6-week $R T$ intervention. The $C G$ did not improve BEV ( $p>0.05)$ following the intervention period. However, the RTG did experience a significant increase in BEV $(p<0.05)$ following the 6-week core $R T$ period. Conclusion: Within the parameters of this study, a 6-week core RT program led to increased BEV among HS baseball players.
\end{abstract}

Keywords: Core stability, core training program, high school baseball, ballexit velocity, hitting

\section{Introduction}

Coaches work together to improve performance and frequently reflect upon questions like, "How should you train a baseball player to hit further? Throw harder? Run faster?" and "How can I best prepare my athlete to compete at the next level?" Players want to know the answers. In baseball, players desire and coaches demand the 5 tools: 1) speed, 2) arm strength, 3) fielding, 4) hitting for average, and 5) hitting for power (Kohmura, Aoki, Yoshigi, Sakuraba, \& Yanagiya, 2008). Players that possess a more complete set of tools are more likely to get recruited, drafted, and financially compensated.

${ }^{ \pm}$Physical and Health Education Instructor \& Baseball Coach, Granger High School, West Valley, Utah, USA.

"Professor, Department of Kinesiology and Outdoor Recreation, Southern Utah University, USA. 
Major League Baseball (MLB) has implemented a tool known as "Statcast" in 2015 to analyze and quantify baseball metrics including aspects of pitching, hitting, fielding, base running, and more (Statcast, 2016). According to MLB news writer Paul Casella (2015), Statcast will change the way fans, coaches, and athletes view the game by providing an immediate statistical analysis for any movement on the field. This innovative technology has enabled analysts of the game to compare players with a greater degree of objectivity and reliability. Although few have access to this state-of-the-art technology, it has influenced the way players train, coaches recruit, and scouts evaluate performance. Meaning, throwing velocity (TV) and ball-exit velocity (BEV) are two current performance measures that may be a better predictor of sports performance outcomes than other traditional statistics such as: batting average, earned-run average, wins, or standardized fitness testing. Designing a resistance training (RT) program that could improve these metrics would appear to be of value to players and coaches.

Core RT has recently received a lot of attention for improving functional movement and athletic performance. However, there is limited research that quantifies the effectiveness core RT has on specific motor skills in sports. The term trunk and core are often used interchangeably and can be defined as the connection between the lower and upper extremities. Kenney, Wilmore, \& Costill (2015) define the core as a group of trunk muscles that surround the spine and abdominal viscera and include the abdominals, gluteals, hip girdle, paraspinals, and other accessory muscles. Stabilizers of the core such as the quadratus lumborum, transversus abdominis, multifidus, and internal oblique collaborate to produce intra-abdominal pressure around the spine, creating stabilization and stiffness of the spinal column (Akuthota, Ferreiro, Moore, \& Fredericson, 2008). The erector spinae, external oblique, and rectus abdominis are the long, superficial muscles of the trunk that are capable of producing a great amount of force. The core is called upon for a variety of movements to create stability and balance in daily living and sports performance. The core is engaged to some degree in Olympic lifts, a variety of squats/lunges, and other major push or pull movements (Hedrick, 2004).

Core strength refers to the force production of the core muscles. It is difficult to assess the strength of the core because the muscles that comprise the core are not suited for maximal load testing (example one repetition maximum). Therefore, dynamic endurance tests such as: trunk curls, partial curl-ups, and sit-ups are administered to assess the endurance of the abdominal muscles and to identify abdominal weaknesses (Heyward, 2010). Core strength as it relates to sports performance is the ability to transfer energy through the core during rapid, discrete motor skills such as: punching, kicking, jumping, throwing, and swinging. According to Kibler, Press, \& Sciascia (2006) core stability is defined as the ability to control the position and motion of the trunk over the pelvis to optimize movement and performance. Albeit, the muscles that compose the trunk are often neglected as a primary agonist in an RT program. Including core-specific exercises in an athlete's RT program is recommended to prevent injury and maximize athletic movement (Hibbs, Thompson, French, Wrigley, \& Spears, 2008). The physiology of the core is very complex because it serves two purposes, 
stabilization and force production in three planes: flexion/extension, lateral trunk flexion, and trunk rotation (Hedrick, 2004). Hibbs et al. (2008) states that there is not one single exercise that activates and challenges all of the core muscles. Kibler et al. (2006) explains that the core musculature plays a critical role in kinetic chain activities by generating and transferring energy from large to small body parts.

McGill (2010) has been performing core RT with athletes and rehabilitating patients and recommends core RT for improving balance, strength, and endurance to prevent injury and maximize performance. McGill \& Karpowicz (2009) identified exercises and progressions that adequately stimulate the trunk such as: curl-ups, birddogs, and side bridges to improve spine stabilization. Medicine ball exercises that emphasize rotational movements improve force production in the transverse plane (Szymanski, Szymanski, Bradford, Schade, \& Pascoe, 2007a).

Kenney et al. (2015) discusses the specificity principle in which a RT program must stress the physiological systems critical for optimal performance in a given sport to achieve desired training adaptations in that sport. Baechle \& Earle (2008) have identified core RT as an assistance exercise, less important for improving sport performance, but rather a common application for injury prevention and rehabilitation. Yet, the results of an EMG analysis of the delivery of a pitch indicated $75-100 \%$ recruitment of the abdominal obliques, abdominal rectus, and lumbar paraspinal muscles contralateral to the pitcher's arm (Watkins et al., 1989). Muscular activation of $75-100 \%$ indicates that muscles of the core play a significant role in trunk rotation and force production during the delivery of a pitch. Shaffer, Jobe, Pink, \& Perry (1993) studied the muscle activity (EMG) during a baseball swing and found that hitting is a sequence of coordinated muscle activity; in which force originates in the hips, energy is transferred through the trunk, and terminates with the arms. Haugen, Haugvad, \& Røstad (2016) searched for scientific publications of RT used in athletic populations and found that many different sports will include core RT, but fail to describe a detailed exercise prescription of the RT. According to Willardson (2007) increasing core stability and strength should be a priority for all sports conditioning programs, but certainly applies to sports that are played on unstable surfaces or may require the athlete to perform skills in unstable body positions.

In baseball arm strength is measured using TV. Coaches should evaluate every players' TV because some defensive positions require more arm strength than others. It is a common misconception that the pitcher has the best arm, when in reality, position players can throw with just as much velocity, if not more. The overhand throwing motion uses a proximal to distal sequence to maximize TV. Hirashima, Kadota, Sakurai, Kudo, \& Ohtsuki, (2002) collected kinematic data during maximal TV trials using electrodes to determine the sequence of muscle contraction during the throwing motion. The researchers found that the external oblique contralateral to the throwing arm contracts prior to the ipsilateral side during the rotational movement of the throw, while the rectus abdominis is most prevalent during the follow through (spinal flexion) phase. Manchado, GarcíaRuiz, Cortell-Tormo, \& Tortosa-Martínez (2017) found that increasing core strength contributed to the development of the kinetic chain and concluded that a 10-week core RT program significantly improved the TV of handball players. 
Although TV is a major component to playing defense in baseball, scoring more runs than the other team is how to win the game. Some batters are known as contact hitters and others as power hitters, but all successful hitters reach base safely (Baseball, 2016). Nonetheless, the aim of all hitters is to hit the ball hard. $\mathrm{BEV}$ is a relatively new term in baseball used to measure how fast the ball is leaving the bat. Meaning, a pitch might enter the strike zone at $145 \mathrm{kph}$ (90 mph), but leave the bat at $160.9 \mathrm{kph}(100 \mathrm{mph})$. According to a Statcast analysis by Posnanski (2017), a positive correlation exists between BEV, batting average, and slugging percentage. This stresses the importance of BEV because the faster the ball leaves the bat, the tougher it is to defend. Kohmura et al. (2008) conducted a correlation analysis from a field test that evaluated batting, fielding, base running, and physical fitness testing, revealing that back strength and medicine ball throwing are related to batting performance. This is consistent with Szymanski et al. (2007b) who reported a significant increase in bat swing velocity due to improvement in angular hip velocity, torso rotational strength, and hand speed. Furthermore, torsional exercises like the hitter's throw are recommended for hitters to enhance the way the body uses the kinetic chain to improve the rate of force development during rotational movements (Szymanski et al., 2007b).

Studies by Shaffer et al. (1993) and Watkins et al. (1989) both suggest a need for core RT in respective baseball training regimens to maximize sport-specific skill outcomes based on the EMG of hitting and throwing. McGill (2010), Hibbs et al., (2008), and Willardson (2007) all agree that core RT should be a part of an athlete's training regimen to optimize performance and minimize injury. Following the principle of specificity, implementing a core RT program that challenges the body in a way that is sport-specific should result in the greatest training adaptations. Hence, the purpose of this study was to design an RT program that targets the muscles of the trunk and core that also meets the demands of hitters, pitchers, and position players to increase force production in torsional movements such as throwing and hitting a baseball. It was hypothesized that high school (HS) baseball players' TV (throwing velocity) and BEV (ball-exit velocity) would increase as a result of a 6-week core RT program.

\section{Methods}

\section{Participants}

The volunteering participants were HS students (ages 14-18) and members of the baseball team at Granger HS, Utah, USA. A University Institutional Review Board approved the study (SUU: IRB approval \#30-112018b) and consent/assent forms prior to any engagement with participants. The participants were informed of the purpose of the study and what the research entailed during orientation. Each participant and guardian gave written consent and assent to participate in the study. The subjects' acknowledged that participation was completely voluntary and that they could withdraw from the study without penalty at any time. 


\section{Instruments and Apparatus}

All of the training and testing took place at Granger HS, Utah, USA. The RT was held in the weight room and all testing was completed in the auxiliary gymnasium. All of the training equipment used in this study such as: medicine balls, resistance bands, and free weights were provided by the HS. Stalker radar guns have been used in many studies, as well as professional baseball stadiums because the technology integrated into each gun has led to the highest performance and accuracy over the last 30 years (Stalker Radar, 2017). Hence, the instrument used to measure pre and post-assessment of TV and BEV was the Stalker Sport II radar gun (Applied Concepts Inc., Plano, TX). Equipment used during testing such as: nets/cages, batting tees, baseballs, and artificial mounds were made available by the HS baseball program. Schutt baseball hitting tees were used in the BEV assessment. Additionally, all of the baseballs used in this study were game balls in accordance with the National Federation of State High School regulation (NFHS).

\section{Procedures}

The participants were randomly assigned in a stratified manner (based on grade) to the control group (CG) or the resistance training group (RTG). The CG practiced Tuesdays and Thursdays from 7-9 P.M. for six weeks. Practice consisted of baseball-specific training only, which included drills for throwing, fielding, pitching, and hitting. The RTG participated in a 6-week RT program after school twice a week, in addition to the aforementioned practice. Prior to and following the training intervention, both groups had their TV and BEV assessed (Figure 1).

Figure 1. Study Timeline. TV= Throwing Velocity. BEV=Ball-exit Velocity

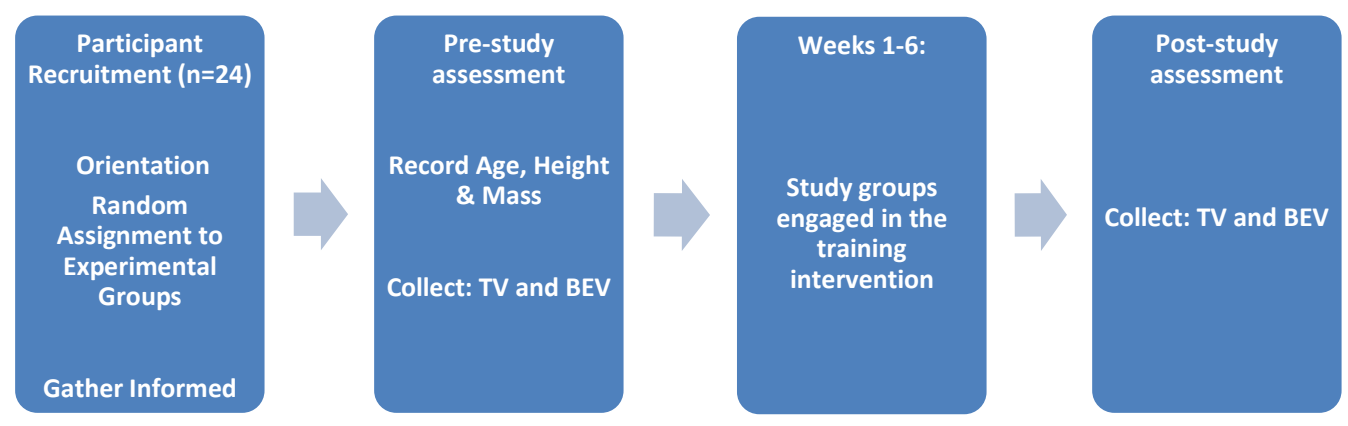

\section{The Training Protocol}

The RTG completed a 6-week core RT program. The RTG met twice per week for one hour in the weight room of Granger HS (Utah, US). Subjects had to attend and participate in 11 of 12 sessions to be included in the study. Each session included a warm-up, the RT, and a cool-down. Table 1 provides the exercises the participants performed twice a week, for six weeks.

The warm-up included a light $400 \mathrm{~m}$ jog, followed by dynamic stretching (knee-to-chest, leg swings, lunges w/ twist). The RT program was broken up into 
seven groups of exercises. The first group of exercises was a series of weighted walks emphasizing core stability. To complete a set of weighted walks, the subjects had to walk $27.43 \mathrm{~m}$ holding dumbbells at approximately $50 \%$ of their body weight; weighted walks with one dumbbell were then performed with $25 \%$ of their body weight. Individuals that could not maintain form with the prescribed weight decreased the weight of the dumbbell and those who could complete all of the sets with proper form increased the weight by $2.27 \mathrm{kgs}$ the following week. The participants were prescribed 1-4 sets, $27.43 \mathrm{~m}$ in distance, for each type of walk (farmer walks, suitcase walks, waiter walks, crossover walks, and zombie walks)(Fig. 2a). Group of exercises 2-5 exposed the participants to a variety of endurance-based movements performed with body weight or dumbbells that challenge the core in different planes. During exercises 2-5, each pair of exercises included 1-3 sets of 12-20 repetitions and were completed as supersets to reduce rest time and to maintain intensity. These exercises include $\mathrm{V}$-sits holds (Figure 2 b), birddogs, iron butterflies, lunges with weighted twists, Romanian deadlifts, abrollouts, back extensions (Figure $3 \mathrm{a}, \mathrm{b}$ ), and hanging leg raises. The 6th group of exercises required a resistance band for the rotational and anti-rotational movements of the Pallof press and the oblique twists. Once the participant was able to complete 3 sets of 12 each side, they could move up to a more difficult resistance band. The last group of exercises (group 7) utilized a 1.81-5.44 kg medicine ball to complete 1-3 sets of the figure 8's and standing medicine ball toss (Figure 4). The subjects completed 8-12 repetitions of the figure 8's and 4-8 repetitions of the standing medicine ball toss, in each direction.

Following the RT, a short cool-down period included static stretching and walking. Immediate individual feedback was provided as necessary and general feedback was provided during the debriefing period at the end of each RT session. For each training session attended, participants completed a workout log provided by the administrator. The main purpose of the workout log was to track personal progression and to follow the exercise sequence.

Table 1. RT Program

\begin{tabular}{|c|c|c|c|c|c|c|c|c|}
\hline $\begin{array}{c}\text { Warm } \\
\text { Up }\end{array}$ & Group 1* & $\begin{array}{c}\text { Group } \\
2 *\end{array}$ & Group 3* & Group 4* & Group 5* & Group 6** & Group 7॰ & $\begin{array}{c}\text { Cool } \\
\text { Down }\end{array}$ \\
\hline $\begin{array}{c}\text { Farmers } \\
\text { walks } \\
\text { meter jog }\end{array}$ & $\begin{array}{c}\text { Suitcase } \\
\text { walks } \\
\text { Waiter } \\
\text { walks }\end{array}$ & $\begin{array}{c}\text { V-sit } \\
\text { holds }\end{array}$ & $\begin{array}{c}\text { Iron } \\
\text { butterflies }\end{array}$ & $\begin{array}{c}\text { Romanian } \\
\text { deadlift }\end{array}$ & $\begin{array}{c}\text { Back } \\
\text { extension }\end{array}$ & $\begin{array}{c}\text { Pallof } \\
\text { press }\end{array}$ & $\begin{array}{c}\text { Figure } \\
8 \text { 's }\end{array}$ & $\begin{array}{c}\text { Statics } \\
\text { Stretching }\end{array}$ \\
\hline $\begin{array}{c}\text { Dynamic } \\
\text { stretching }\end{array}$ & $\begin{array}{c}\text { Zossover } \\
\text { Zalks } \\
\text { Walks }\end{array}$ & Bird & $\begin{array}{c}\text { Longes } \\
\text { Wh } \\
\text { weighted } \\
\text { twist }\end{array}$ & $\begin{array}{c}\text { Ab- } \\
\text { Rollouts }\end{array}$ & $\begin{array}{c}\text { Hanging } \\
\text { leg raises }\end{array}$ & $\begin{array}{c}\text { Oblique } \\
\text { twists w/ } \\
\text { bands }\end{array}$ & $\begin{array}{c}\text { Standing } \\
\text { medicine } \\
\text { ball toss }\end{array}$ & Walking \\
\hline
\end{tabular}

Note: *Body weights or dumbbells utilized. +Resistance bands utilized. $\odot$ Medicine balls utilized. 
Figure 2. (a) Cross over Walk (b) V-sit Hold

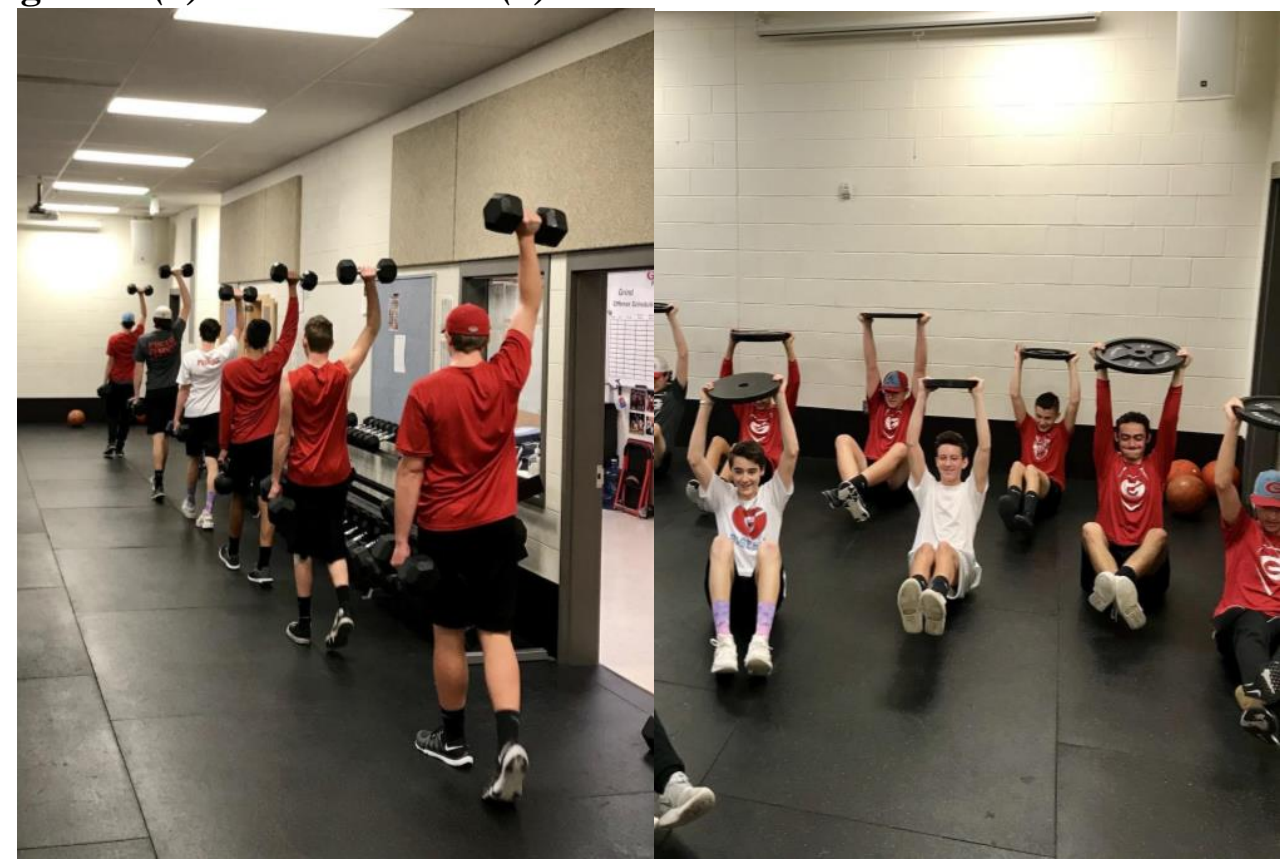

(a)

(b)

Figure 3. Back Extension (a) Flexed (b) Extension

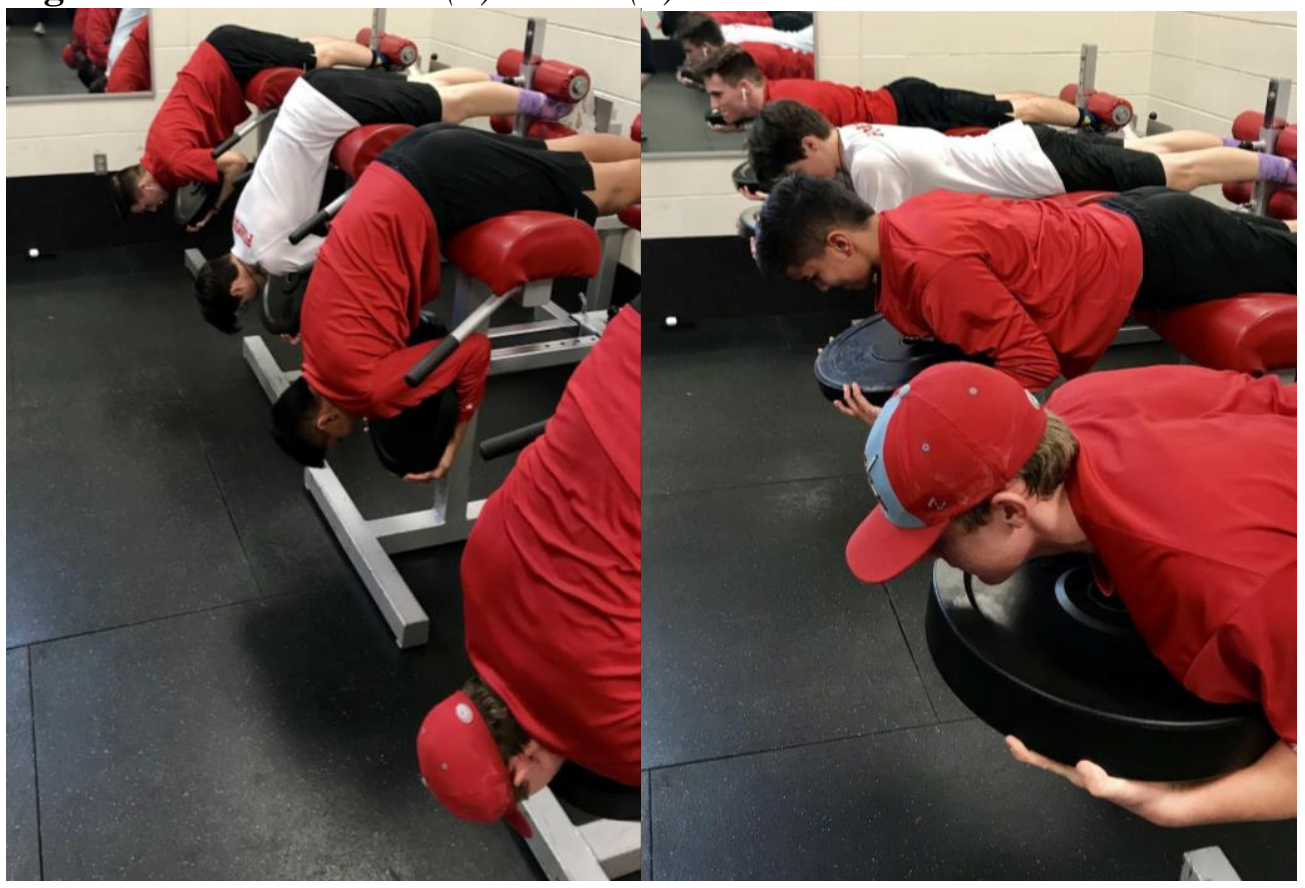

(a)

(b) 
Figure 4. Standing Medicine Ball Toss (a) Load (b) Explode

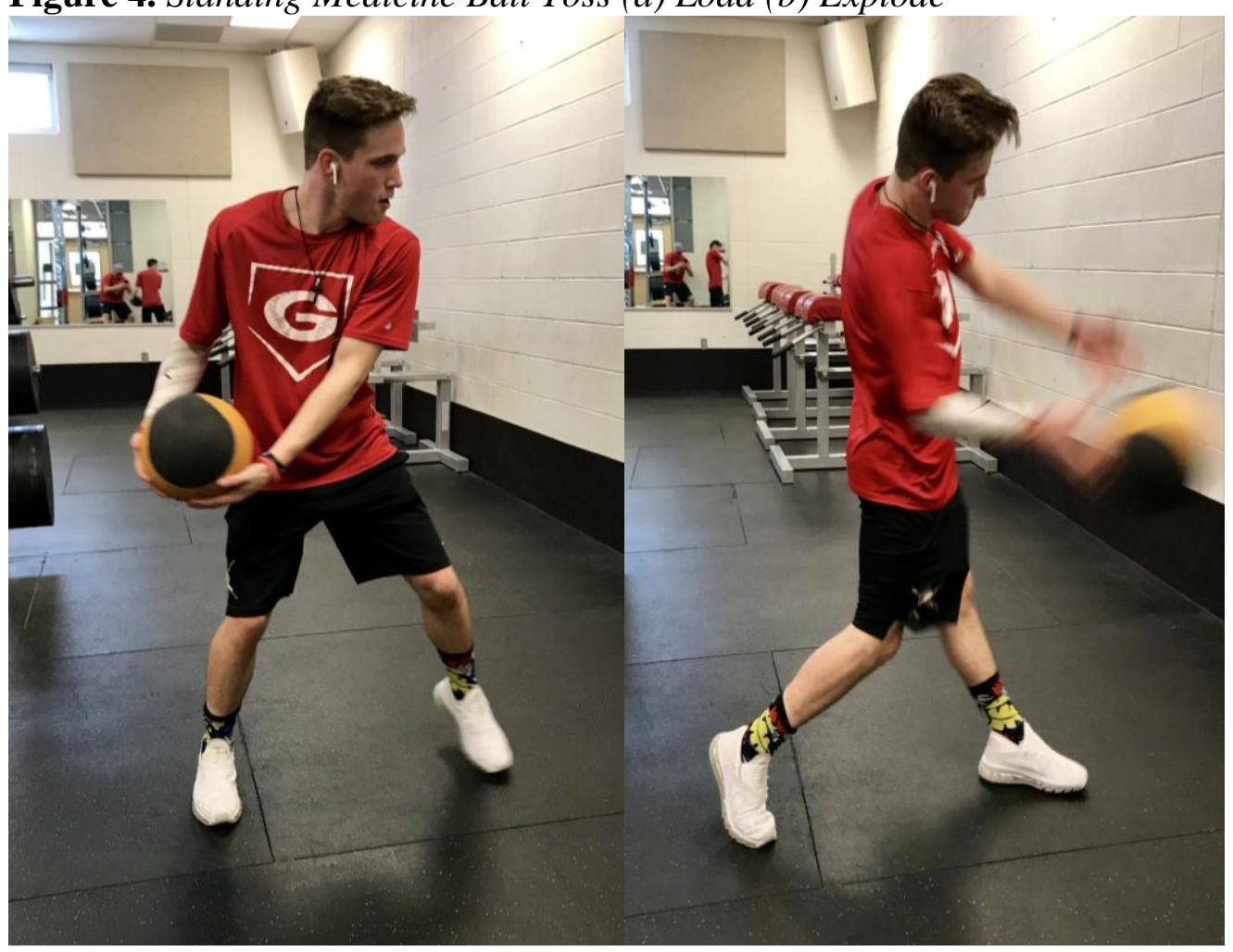

(a)

(b)

The Testing Protocol

The data collection process and procedures for the pre and post-test were administered in the same manner. It took 2 days to collect the baseball team's TV and BEV trials (Figure 5). A baseball specific warm-up was included prior to each day of testing. All participants warmed-up with a short-jog, followed by a sequence of dynamic stretching, and finished by playing catch with a partner.

Day 1, all subjects, regardless of position made 4 throws across the gym to a catcher to measure TV. The distance of the throw was $27.43 \mathrm{~m}$ as indicated by a tape marking on the floor. A ground ball was rolled to the players and using appropriate footwork such as a "crow's hop" the participants fielded the ball and then delivered the ball with maximum TV to the catcher. Subjects rotated after each trial to provide adequate recovery time between throws. The radar gun was operated from behind the catcher. The $4 \mathrm{TV}$ trials were recorded directly from the radar gun display into an Excel spreadsheet. The average of the $4 \mathrm{TV}$ trials was used for statistical analysis. 
Figure 5. Testing Protocol Timeline. TV= Throwing Velocity. BEV= Ball-exit Velocity

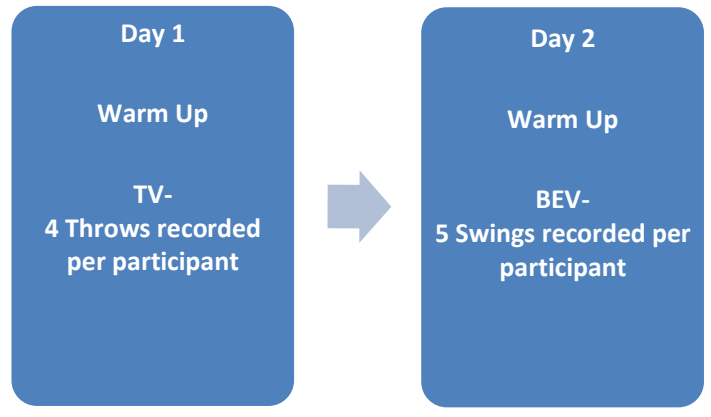

Day 2 the BEV was assessed by hitting the ball off a batting tee and into a net. $\mathrm{BEV}$ is most valid and reliable when the ball is moving directly at or away from the radar gun. Therefore, the radar gun was stationed behind the batting tee (in the catcher's position) at pitch height (Figure $6 \mathrm{a}, \mathrm{b}$ ). Hitters took 5 swings off of the tee and the velocities were recorded and averaged for statistical. The subjects were required to hit the ball towards centerfield for the swing to count. The subjects were allowed to use their own bat for the BEV test, but it had to meet NFHS regulation (BBCOR .50, psi, barrel size, and length-weight ratio). Batters adjusted the Schutt hitting tee to their preferred height to maximize BEV.

Figure 6. Ball-exit Velocity Assessment (a) Load (b) Explode

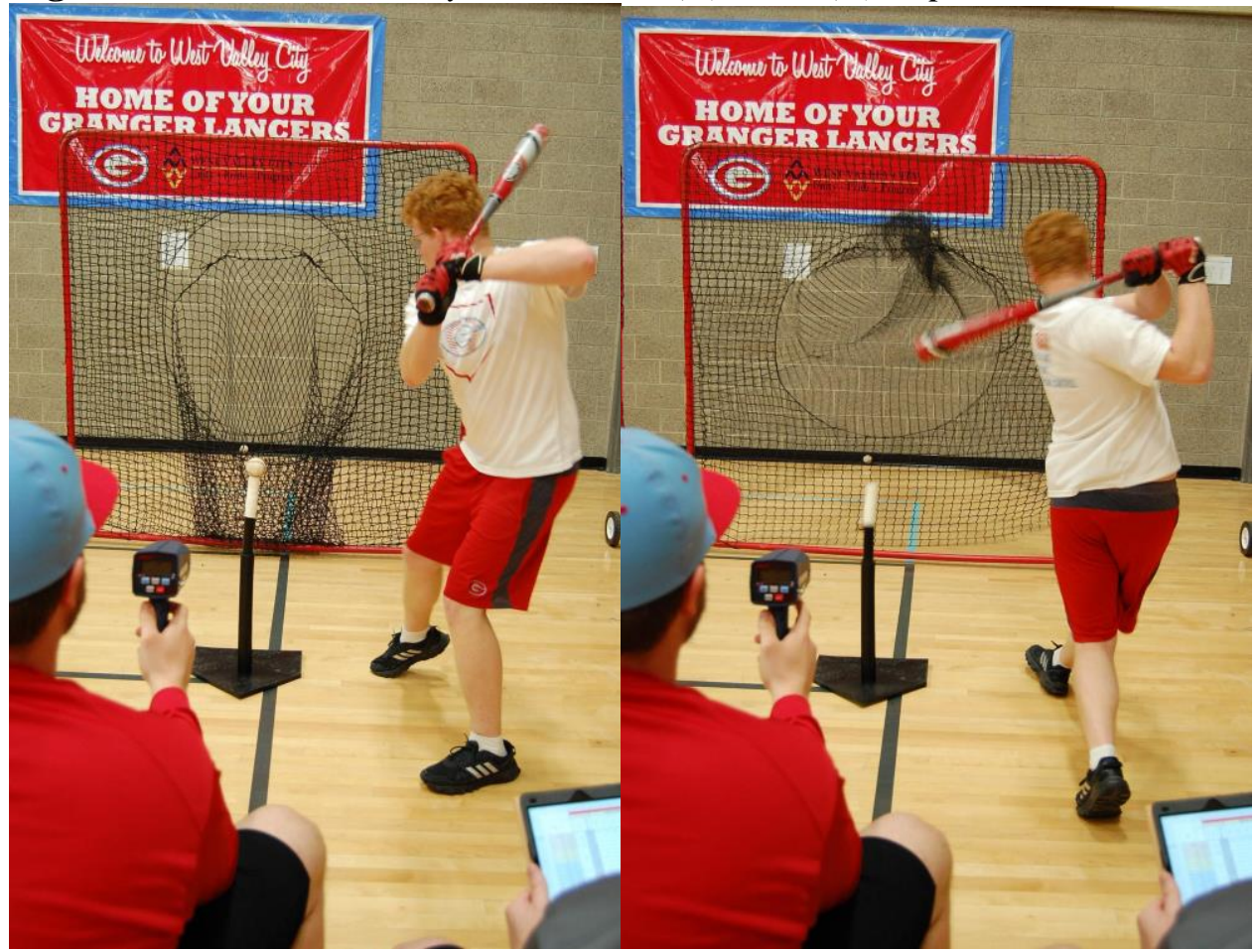

(a)

(b) 


\section{Reliability}

The Stalker Sport II radar gun was the instrument selected to collect TV and BEV. During testing, the Stalker Sport II radar gun was set to follow the recommended settings for baseball scouts found in the owner's manual, which included a low speed of $48.28 \mathrm{kph}(30 \mathrm{mph}$ ) and range setting of 3 (maximum sensitivity). Additionally, the radar gun was stationed directly in front or behind the ball to eliminate angle errors. Stalker radar guns have been reported to measure within 0.10 (one-tenth) of a mile per hour (Stalker Radar, 2017). Harasin, Dizdar, \& Marković (2006) examined the reliability of a Doppler radar gun and reported the intraclass reliability coefficient of ICC $=0.97$.

\section{Design and Analysis}

The TV and BEV were the dependent variables (DVs) measured. The DVs were compared prior to and following the study intervention period within each experimental group using dependent t-tests. A gain score was also calculated for each DV and compared between experimental groups with independent t-tests. Statistics were recorded and analyzed using MS Excel 2013. The Excel spreadsheet of data was peer reviewed for accuracy per Al Tarawneh and Thorne (2017). The statistical significance for the study was $\alpha<0.05$.

\section{Results}

There were 24 HS male baseball players between 14-18 years old that participated in this study. All participants adhered to and completed the study without complication. Descriptive participant information can be found in Table 2 .

Table 2. Participation Descriptive Information

\begin{tabular}{|l|c|c|c|c|}
\hline & Number & Age (years) & Height $(\mathrm{cms})$ & Mass $(\mathrm{kg})$ \\
\hline RTG & 12 & $15.4 \pm 1.1$ & $170.8 \pm 8.4$ & $74.6 \pm 19.2$ \\
\hline CG & 12 & $15.6 \pm 1.3$ & $172.5 \pm 7.8$ & $75.8 \pm 21.8$ \\
\hline
\end{tabular}

Mean \pm standard deviation. $\mathrm{RTG}=$ Resistance Training Group. $\mathrm{CG}=$ Control Group

Neither experimental group statistically improved TV following the 6-week study intervention period ( $p>0.05$ ). Likewise, the CG did not improve BEV following the 6-week study intervention period $(p>0.05)$. However, BEV significantly improved $(\mathrm{p}<0.05)$ in pre to post-assessment in the RTG. The gain score for the BEV was significantly greater for the RTG than the CG (see Table 3 ). Note that Table 3 is in units of miles per hour, as that is the standard in baseball. 
Table 3. Throwing Velocity and Ball-Exit Velocity (MPH)

\begin{tabular}{|l|c|c|c|c|c|c|}
\hline \multicolumn{5}{|c}{ TV } & \multicolumn{3}{c|}{ BEV } \\
\hline & Pre-Ave. & Post-Ave. & Gain & Pre-Ave. & Post-Ave. & Gain \\
\hline RTG & $64.5 \pm 4.5$ & $65.2 \pm 4.0$ & $0.6 \pm 1.9$ & $69.7 \pm 6.6$ & $72.8 \pm 5.8^{+}$ & $3.1 \pm 2.3^{*}$ \\
\hline CG & $59.3 \pm 8.2$ & $60.2 \pm 6.3$ & $1.0 \pm 3.4$ & $66.4 \pm 7.9$ & $67.7 \pm 7.3$ & $1.2 \pm 3.2$ \\
\hline
\end{tabular}

Means \pm standard deviation for dependent variables. TV= Throwing Velocity. BEV=Ball-Exit Velocity. RTG= Resistance Training Group. $\mathrm{CG}=$ Control Group. + Significant improvement pre to post intervention within the RTG $\mathrm{p}<0.05$. MPH-miles per hour. *RTG BEV gain score significantly greater than the $\mathrm{CG}(\mathrm{p}<0.05)$.

\section{Discussion}

The purpose of this study was to design a RT program that targets the musculature of the core that also meets the demands of hitters, pitchers, and position players to increase force production in torsional movements. The intent of the RT program was to improve core stability and strength using free weights, resistance bands, and medicine balls to increase TV and BEV of HS baseball players. A primary objective of this study was to improve sport-specific training methods to peak baseball performance. It was hypothesized that the HS baseball players' TV and BEV would increase as a result of the 6-week core RT program.

The results of the study were mixed with respect to the research hypothesis. Both groups on average improved TV and BEV from pre to post-assessment, however, only the BEV for the RTG demonstrated a significant improvement from pre to post-assessment $(\mathrm{p}<0.05)$. The subjects within the RTG improved their BEV by $4.99 \mathrm{kph}$ (3.1 mph) following the 6-week core RT program. The RTG's BEV gain score was almost significantly greater than the CG's BEV $(p<0.056)$. The difference in gain scores from the RTG to the CG was $3.06 \mathrm{kph}(1.9 \mathrm{mph})$. Meaning, on average players hit the ball approximately significantly harder than the CG following the 6-week RT training intervention. Posnanski (2017) claims good hitting in the MLB begins with a BEV of $148 \mathrm{kph}(92 \mathrm{mph})$ and suggested that an increase of $1.61 \mathrm{kph}(1 \mathrm{mph})$ in BEV would increase a hitter's batting average by .025 and slugging percentage by 0.050 . Assuming Posnanski's (2017) postulate is true, the results of the current study suggest that implementing a similar core based RT program could improve a hitter's batting average and slugging percentage in a very meaningful manner. Based on Posnanski's postulate, a hitter could expect an increase in batting average of 0.048 and slugging percentage of 0.095 by engaging in a similar core RT program. As a team, this increase in hitting performance could contribute to a more productive scoring offense leading to more wins.

Previous research related to hitting in baseball has focused on the: biomechanics of a baseball swing (Shaffer et al., 1993; Welch, Banks, Cook, \& Draovitch, 1995), the change in bat speed after swinging over and underweight bats (DeRenne, Buxton, Hetzler, \& Ho, 1995), and the effect of specific RT programs on bat swing velocity (Hughes, Lyons, \& Mayo, 2004); none of which report on $\mathrm{BEV}$. There is little data specifically related to BEV in baseball because it is a relatively new term and has not been investigated. As such, the data collected in 
the current study cannot be compared to a normative datum. Closely related research has focused on bat speed as the variable of interest. Szymanski, DeRenne, \& Spaniol (2009) reviewed prior research that focused on RT as related to improving bat swing velocity and found torso rotational strength to be a significant performance variable in hitting. Although bat swing velocity and BEV are two different variables, hitters should be trained in the same way because bat swing velocity creates $\mathrm{BEV}$. Implementing a RT program that improves torso rotational strength should increase bat swing velocity, presumably leading to a higher BEV. Per Posnanski's postulate, greater BEV should lead to an improved batting average and slugging percentage.

The TV gains of the RTG and CG showed no significant improvement based on a 6-week study intervention. Our findings are consistent with Newton \& McEvoy (1994) who did not improve TV with a medicine ball training intervention that prescribed dynamic exercises such as: chest pass, overhead throws, and medicine ball toss. Van Den Tillaar's (2004) review categorized various RT studies (RT with 3x6 RM, RT with Pyramid scheme, RT between 8-12 RM, RT lower than $12 \mathrm{RM}$ ) related to the velocity of overarm throwing and reported that various forms of RT modalities such as: bench press, throwing overweight/ underweight baseballs, throws with a pulley device, and medicine ball training could improve TV. More specifically, Van Den Tillaar (2004) concluded that RT consisting of 3 sets of 6-RM or between 8 and 12-RM (bench press) lead to significant increases in TV and that medicine ball training did not positively improve TV. More recently, Manchado et al. (2017) found that a 10-week core RT program increased TV of handball players. Even though the subjects were handball players, the exercises prescribed (curl-up with twist and frontal bridge with Swiss ball) focused on strengthening the lumbo-pelvic region to maximize the efficacy of the overhand throw; similar to our study that prescribed dynamic and static movements such as: V-sit holds, ab-rollouts, hanging leg raises, etc. Although there are inconsistencies in RT prescription for improving $\mathrm{TV}$, the muscle recruitment pattern (EMG) recorded during the delivery of a pitch explains the sequential muscle activity of the trunk during overhand throwing, which indicates that training the core should be advantageous for throwers (Watkins et al., 1989; Hirashima, 2002). Hedrick (2004) claims the trunk is the most important muscle group to train in order to improve athletic performance because the muscle actions of the core provide stabilization and force/power production. Likewise, Hibbs (2008) states there is not one single exercise that activates and challenges the entire musculature of the core. Hence, it seems that the optimal method of RT for increasing TV is yet to be determined, but it appears core exercises should be included.

Muscle action and muscle group specificity were two current concepts defined by Fleck \& Kraemer (2014) that contributed to the design of the core RT program used in the current study. In accordance with the specificity principle, the closer training is to the sport, the greater chance there will be a positive transfer to that specific sport (Kenney et al. 2015). Although the muscles of the core are involved during a swing and a throw, the mechanics of each movement differ. The rotational exercises (Lunge w/ weighted twist, oblique twists with resistance 
bands, and standing medicine ball toss) selected for this RT program were designed to maximize force production during a baseball swing. For example, the standing medicine ball toss looks and feels like an at-bat, but provides athletes with an opportunity to practice using the kinetic chain by transferring energy sequentially from the lower body, through the core, and to the hands. The kinetic chain theory is supported by Kibler et al. (2006) who believes that the muscles of the core link the body together to create a kinetic chain capable of producing significant amounts of force and power. The RTG's gain score for BEV is likely due to the rotational exercises performed with resistance bands and medicine balls, and is supported by the kinetic chain concept and specificity principle. As previously stated, the throwing motion is a complex series of coordinated muscle contractions, requiring movement in the transverse and sagittal plane. The lack of significant improvement in TV could be due to the unique biomechanics of throwing a baseball. Additionally, our program included stabilization and flexion/ extension exercises (weighted walks, birddogs, back extensions, etc.) that addressed all phases of an overhand throw, but the RT may not have been similar enough to the overhand throwing motion to increase TV. Whereas, DeRenne, Buxton, Hetzler, \& Ho (1994) significantly improved TV by throwing with a combination of standard, light, and heavy baseballs 3 days a week for 10 weeks.

The primary limitations in this study were sample size, population, and experience. For the TV and BEV assessments the sample size was 24 studentathletes which was limited by the number of baseball players at Granger HS, UT. Additionally, student-athletes aged 14-18 were a challenging population to work with. Among this age group, lack of time and availability were limiting factors when designing the RT program to fit the schedule of the student-athletes. Despite the fact that all the students completed the program, uncontrollable factors could have hindered sports performance such as nutrition, sleep, or missed practice. Another limitation relates to the experience of the athletes. All of the subjects in the study were amateur and their previous level of fitness varied. All athletes had continuous exposure to baseball practice and it is possible some athletes had considerable improvement in TV and BEV unrelated to the RT provided. Finally, a longer intervention period may have yielded statistical differences as related to the dependent variables.

Another point of interest regarding the current study is the use of a batting tee for the purpose of collecting the BEV. The batting tee is one of the most common fundamental training tools used for developing a mechanically sound baseball swing. Coinciding with Newton's 2nd law of motion, the BEV off of a batting tee will only have the velocity of the bat swing, whereas the BEV of a batted-ball from a live pitch will have a resulting velocity of the pitched ball and the swing. Meaning, a potential limitation of using the tee to measure BEV is that peak BEV will be lower off of a tee than a pitched ball. However, measuring BEV off a tee, rather than a live pitch is more reliable because the ball is not moving and the hitter determines the location of the collision between the bat and ball. Hitting the ball off of the tee creates a closed environment for the hitter, though different from live pitching, it trains hitters to create maximal force production upon a motionless baseball using proper form. This is likely to carry over to hitting performance in a 
game because the hitter can produce higher quality swings as a result of training off a batting tee.

Moving forward, researchers should compare core RT to other methods of RT to help shape the most appropriate RT program for baseball players; especially since the experimental and clinical data are limited in this population. Future research should report BEV and TV in order to define an appropriate normative velocity scale for each age group in baseball. Additionally, gathering TV and BEV data could be useful in terms of selecting players to make the team each season, tracking player development, or even determine who the next best recruit may be.

\section{Conclusion}

This study implemented a 6-week RT program that focused on the development of the core musculature to directly improve baseball performance. Within the parameters of this study, a core RT program led to an increase in BEV among HS baseball players. A secondary objective of this study was to identify sport-specific training methods to peak baseball performance and we have unlocked significance in core RT specifically related to BEV. Implementing additional rotational exercises that utilize free weights, resistance bands, or medicine balls may lead to additional gains in torso rotational strength and potentially greater improvements in BEV.

\section{References}

Akuthota, V., Ferreiro, A., Moore, T., \& Fredericson, M. (2008). Core stability exercise principles. Current Sports Medicine Reports, 7(1), 39-44.

AlTarawneh, G., \& Thorne, S. (2017). A pilot study exploring spreadsheet risk in scientific research. arXiv preprint arXiv:1703.09785. arvix.org, Ithca, NY.

Baseball. (2016, May 13). New World Encyclopedia. Retrieved June 28, 2019 from// www.newworldencyclopedia.org/p/index.php?title=Baseball\&oldid=995930.

Casella, P. (2015, April \& may). Statcast primer: Baseball will never be the same. Retrieved December 12, 2018, from https://www.mlb.com/news/statcast-primer-ba seball-will-never-be-the-same/c-119234412

DeRenne, C., Buxton, B.P., Hetzler, R.., \& Ho, KW. (1994). Effects of under-and overweighted implement training on pitching velocity. Journal of Strength \& Conditioning Research, 8(4), 247-250.

DeRenne, C., Buxton, B.P., Hetzler, R.K., \& Ho, K.W. (1995). Effects of weighted bat implement training on bat swing velocity. Journal of Strength \& Conditioning Research, 9(4), 247-250.

Fleck, S.J., \& Kraemer, W.J. (2014). Designing Resistance Training Programs (4th ed.). Champaign, IL: Human Kinetics.

Harasin, D., Dizdar, D., \& Marković, G. (2006). High reliability of tests of maximum throwing performance. Journal of Human Movement Studies, 51(1), 63-76.

Haugen, T., Haugvad, L., \& Røstad, V. (2016). Effects of core-stability training on performance and injuries in competitive athletes. Sportscience, 20, 1-7.

Hibbs, A.E., Thompson, K.G., French, D., Wrigley, A., \& Spears, I. (2008). Optimizing 
performance by improving core stability and core strength. Sports Medicine, 38(12), 995-1008.

Hirashima, M., Kadota, H., Sakurai, S., Kudo, K., \& Ohtsuki, T. (2002). Sequential muscle activity and its functional role in the upper extremity and trunk during overarm throwing. Journal of Sports Sciences, 20(4), 301-310.

Hedrick, A. (2004). Learning from each other: Training the trunk. Strength \& Conditioning Journal, 26(6), 70.

Heyward, V.H. (2010). Advanced Fitness Assessment and Exercise Prescription (6th ed.). Champaign, IL: Human Kinetics.

Hughes, S.S., Lyons, B.C., \& Mayo, J.J. (2004). Effect of grip strength and grip strengthening exercises on instantaneous bat velocity of collegiate baseball players. Journal of Strength \& Conditioning Research, 18(2), 298-301.

Kohmura, Y., Aoki, K., Yoshigi, H., Sakuraba, K., \& Yanagiya, T. (2008). Development of a baseball-specific battery of tests and testing protocol for college baseball players. Journal of Strength \& Conditioning Research, 22(4), 1051-1058.

Kenney, W.L., Wilmore, J., \& Costill, D. (2015). Physiology of Sport and Exercise (5th ed.). Champaign, IL: Human Kinetics.

Kibler, W.B., Press, J., \& Sciascia, A. (2006). The role of core stability in athletic function. Sports Medicine, 36(3), 189-198.

Manchado, C., García-Ruiz, J., Cortell-Tormo, J.M., \& Tortosa-Martínez, J. (2017). Effect of core training on male handball players' throwing velocity. Journal of Human Kinetics, 56(1), 177-185.

McGill, S. (2010). Core training: Evidence translating to better performance and injury prevention. Strength \& Conditioning Journal, 32(3), 33-46.

McGill, S., \& Karpowicz, A. (2009). Exercises for spine stabilization: Motion/motor patterns, stability progressions, and clinical technique. Archives of Physical Medicine \& Rehabilitation, 90(1), 118-126.

Posnanski, J. (2017, May 20). Fun with statcast ${ }^{\mathrm{TM}}$ (exit velo). Joe Blogs. Retrieved July 10, 2019 from https://medium.com/joeblogs/fun-with-statcast-exit-velo-20e4ec314 744

Shaffer, B., Jobe, F.W., Pink, M., \& Perry, J. (1993). Baseball batting: An electromyographic study. Clinical Orthopaedics \& Related Research, (292), 285-293.

Stalker Radar. Designs high quality electronics and is the nation's largest manufacturer of speed radar. (n.d.). Retrieved December 12, 2017, from http://www.stalkerradar. com/sportsradar/about.html

Statcast | Glossary. (2016, January 20). Retrieved December 12, 2017, from http://m.mlb. $\mathrm{com} / \mathrm{glossary} /$ statcast

Szymanski, D.J., Szymanski, J.M., Bradford, T.J., Schade, R.L., \& Pascoe, D.D. (2007a). Effect of twelve weeks of medicine ball training on high school baseball players. Journal of Strength \& Conditioning Research, 21(3), 894.

Szymanski, D.J., McIntyre, J.S., Szymanski, J.M., Bradford, T.J., Schade, R.L., Madsen, N.H., \& Pascoe, D.D. (2007b). Effect of torso rotational strength on angular hip, angular shoulder, and linear bat velocities of high school baseball players. Journal of Strength \& Conditioning Research, 21(4), 1117-1125.

Szymanski, D.J., DeRenne, C., \& Spaniol, F.J. (2009). Contributing factors for increased bat swing velocity. Journal of Strength \& Conditioning Research, 23(4), 1338-1352.

Van Den Tillaar, R. (2004). Effect of different training programs on the velocity of overarm throwing: a brief review. Journal of Strength \& Conditioning Research, $18(2), 388-396$.

Watkins, R.G., Dennis, S., Dillin, W.H., Schnebel, B., Schneiderman, G., Jobe, F., \& Pink, M. (1989). Dynamic EMG analysis of torque transfer in professional baseball 
pitchers. Spine, 14(4), 404-408.

Welch, C.M., Banks, S.A., Cook, F.F., \& Draovitch, P. (1995). Hitting a baseball: A biomechanical description. Journal of Orthopaedic \& Sports Physical Therapy, 22(5), 193-201.

Willardson, J.M. (2007). Core stability training: Applications to sports conditioning programs. Journal of Strength \& Conditioning Research, 21(3), 979-985. 